\title{
Revision after failed discectomy
}

\author{
Christoph Mehren ${ }^{1,2} \circledast$ Lorenz Wanke-Jellinek $^{1,2} \cdot$ Andreas Korge $^{1,2}$
}

Received: 31 May 2019 / Revised: 31 May 2019 / Accepted: 13 October 2019 / Published online: 29 October 2019

(C) The Author(s) 2019

\begin{abstract}
Purpose Recurrent lumbar disc herniation is the most common complication after discectomy. Due to the altered anatomy with the presence of scar tissue, the surgical revision of already operated patients could be a surgical challenge.

Methods We describe the microsurgical revision technique step by step with the evaluation of our own clinical results in comparison with primary lumbar disc surgeries. The clinical data are based on a clinical register with 2576 recorded primary surgeries (PD) and 592 cases of revisions (RD) with 12- and 24-month follow-up (FU). The intraoperative dura lesion rates of the surgeries between 2016 and 2018 were recorded retrospectively. Data from 894 primary disc surgeries and 117 revisions were evaluated.

Results The ODI and the VAS for leg and back pain improved in both groups significantly with slightly inferior outcome of the revision group. The ODI improved from 46.3 (PD) and 45.9 (RD), respectively, to 12.6 (PD) and 22.9 (RD) at the 24-month FU. The VAS dropped down as well in both group [VAS back: 47.8 (PD) and 43.9 (RD) to 19.9 and 32.2 at the 24-month FU; VAS leg: 62.9 (PD) and 65.5 (RD) to 15.6 and 26.8 at the 24-month FU]. During the primary interventions, we observed $1.5 \%$ (11/894) and during revisions $7.7 \%$ (9/117) of dura lesions.
\end{abstract}

Conclusions There is no clear guideline for the surgical treatment of recurrent disc herniations. In most cases, a pure rediscectomy is sufficient and can be performed safely and effectively with the help of a microscope.

\section{Graphic abstract}

These slides can be retrieved under Electronic Supplementary Material.
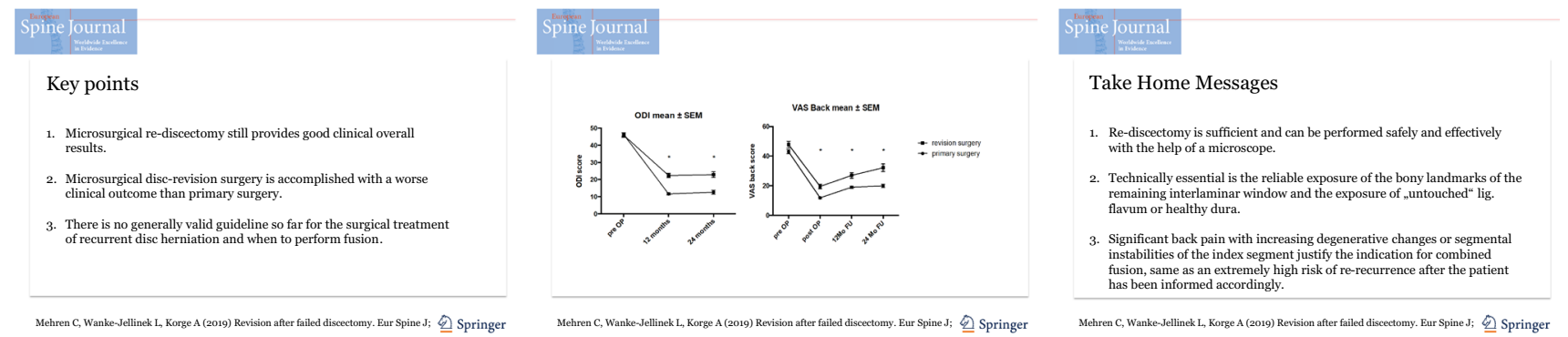

Keywords Recurrent lumbar disc herniation - Operative strategies in lumbar revision - Clinical results after lumbar discectomy and re-discectomy

Electronic supplementary material The online version of this article (https://doi.org/10.1007/s00586-019-06194-9) contains supplementary material, which is available to authorized users.

Christoph Mehren

cmehren@schoen-kliniken.de

1 Spine Center, Schön Klinik München Harlaching, Harlachinger Strasse 51, 81547 Munich, Germany

2 Academic Teaching Hospital and Spine Research Institute, Paracelsus Private Medical University, Salzburg, Austria

\section{Introduction}

Surgical treatment of herniated discs usually provides immediate and very satisfactory results [1]. Even though today minimally invasive methods have prevailed over open discectomy, studies to date have not shown any relevant medium and long-term effects [2,3]. Nevertheless, microsurgical or endoscopic discectomy has established itself as 
the standard procedure. Despite the small minimally invasive accesses, scar tissue still forms in the access area and in the spinal canal. This can cause problems especially for inexperienced surgeons due to the changed anatomy and adherence.

Recurrence of intervertebral discs or bony re-stenosis is frequent indication for revision after previous intervertebral disc operations. However, irrespective of the surgical technique, the incidence of recurrent disc herniation still varies in the literature significantly due to different follow-up durations, diagnostic tools, the definition of recurrency (Is a disc herniation at the same level on the other side a "recurrent disc herniation ?") and individual decision making process when to perform an MRI. Lebow et al. could show that in routinely performed MRIs 2 years after discectomy $56 \%$ of the patients had asymptomatic disc reherniations [4].

Nevertheless, we as surgeons should be aware of the fact that every surgical intervention-regardless of the technique and invasivity-is a "palliative treatment" regarding the disc health status. Up to now, we are not able to prevent revision surgery for sure, and therefore, we have to know the surgical indications and strategies for revisions after failed discectomy.

\section{Materials and methods}

A retrospective analysis of the basic documentation (VAS leg and back, ODI and subjective patient satisfaction), which was prospective collected in a hospital-owned register, was evaluated with regard to the own clinical results. A total of 2576 patients were operated on in our center (between 2011 and 2018) primarily on an intervertebral disc herniation on the lumbar spine (PD); in 592 cases, a revision of an intervertebral disc operation without fusion was performed (RD). Only complete data sets at the respective follow-up (FU) (pre-op, 12 and 24 months post-op) were evaluated.

In order to be able to make a statement about the iatrogenic dura lesion during primary interventions in comparison with revisions, the disc operations of the years 2016-2018 were retrospectively reviewed with regard to intraoperative dura lesions. During these 3 years, 894 primary discectomies and 117 revisions of recurrent disc herniations were performed.

\section{Indications}

Consensus is lacking regarding optimal surgical treatment of a recurrent lumbar disc herniation. The range of intraoperative treatment options extends from decompression only to the final fusion of the index segment. Although fusion surgeries eliminated re-recurrence of the disc herniation, this coincided with additional complication possibilities as well as biomechanical iatrogenic changes, especially in the adjacent segment.

Regardless of the number of previous operations, first of all the clinical symptoms should carefully be evaluated. As our data will also show, a leading radiculopathy can be expected to lead to a significant and sustained improvement in postoperative symptoms, with a significant reduction in back pain as well with a non-instrumented microsurgical technique. After the exclusion of a significant instability on the basis of a further radiological evaluation ( $\mathrm{x}$-ray in 2 plans and possibly with flex/ext), the indication for a isolated revision of the recurrent disc herniation is given.

The complication risk profiles of decompression surgeries and fusion surgeries must be balanced with the individual clinical symptoms, the potential risk of recurrence as well as the individual patients expectations.

\section{Surgical technique}

The previous surgical technique influences the revision strategy. In contrast to the first operation, the surgeons are faced with scar tissue and altered anatomical landmarks in terms of bony defects. In principle, all operative techniques (endoscopic, micro- and macrosurgical with and without fusion procedures) are also evaluated in revision surgery. The selection of the appropriate technique should be adapted to the surgeon's experience. In most cases, the revision with the support of the microscope as standard has proven to be a safe and effective option in our center. In the following, the steps of microsurgical re-discectomy/re-decompression will be described.

The exact localization of the recurrent disc herniation should be known as well as its topographic relation to the nerve root and/or scar tissue. This can be achieved best with an MRI (if necessary with contrast media). The bony landmarks (e.g., medial facet border, lamina border, isthmus) can be seen on the x-rays of the lumbar spine. If necessary, a CT scan can give detailed information about the extension of the previous laminotomy, hemilaminectomy, facetectomy, etc. It also gives information about a potential ossification of the disc herniation.

The disc space height is localized under fluoroscopic control, and a $1.5-2 \mathrm{~cm}$ small skin incision is placed centered over the disc space or over the maximum extension of the recurrency. Sharp subperiosteal dissection is done preferably from the remaining superior lamina down to the transition zone of the superior lamina and the inferior facet. Essential is the reliable exposure of the bony "landmarks." At this point, the scar tissue can be safely detached from the bony rim of the lamina and the medial border of the inferior facet. Care has to be taken if the MRI shows a bulging of the dura dorsal to the lamina border. With a small blunt dissector or a diamond drill, the caudal border of the superior lamina 
is undercut until untouched lig. flavum or healthy dura is exposed sublaminar. Once healthy dura is identified, dissection of the scar tissue is started along the medial border of the inferior facet until the rim of the superior facet is identified. Blunt dissection is performed between bone and scar until you can expose the lateral border of the exiting nerve root followed by decompression along the shoulder of the nerve root till you reach the caudal pedicle. In case of significant fibrosis, a layer of scar tissue is left on the nerve root, respectively, on the dural sac to avoid dural tears.

The exposure of the lateral dural margin, respectively, of the lateral nerve root margin is followed by a careful mobilization of the root to the middle. There are often adherences of the nerve root with the disc space. In these cases, it is advisable to leave the nerve root in place and open the scar tissue lateral to the nerve root to get a safe access to the recurrent herniation. The herniated disc can then be mobilized carefully with a blunt dissector or with a small nerve hook.

At the end of the operation, the neural structures, especially the dura, are checked again for integrity and sufficient decompression. Careful hemostasis and irrigation of the approach and the epidural space finalize the intervention.

This procedure should also be carried out in detail in the same way if the re-discectomy is combined with a fusion of the corresponding segment.

\section{Results}

Regarding the ODI, no significant difference between the primary operations and the revisions could be observed preoperatively in the available data sets. The mean ODI value of the primary operations was 46.3 and 45.9 for the revisions. In the 12- and 24-month follow-up, respectively, there was a highly significant difference between these groups with 11.6 and 12.6 for the PD group and 22.4 and 22.9 for the $\mathrm{RD}$ group, respectively, but even the postoperative results of the RD group improved significantly $(p<0.05)$ compared to

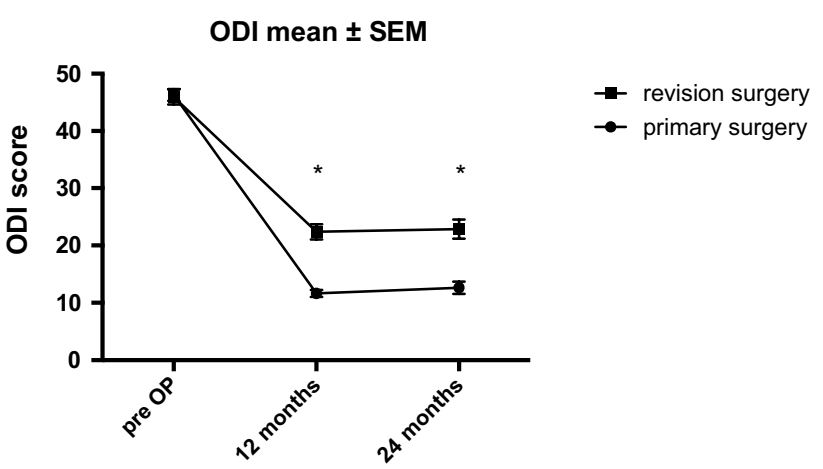

Fig. 1 Preoperative and postoperative course of the ODI for primary versus revision surgery up to 24 month follow up the preoperative values. These results are shown graphically in Fig. 1.

A similar picture emerged for the VAS leg and back pain. Although slightly higher values in the RD group (47.8 vs 43.9) were given preoperatively in mean for VAS back, statistically no corresponding significance was found. However, this was found to be highly significant in the further FU. In the PD group, a clear reduction of the VAS back in the FU could be observed (19.0 in the 12-month FU and 19.9 in the 24-month FU). This is also true for the RD group (27.0 resp. 32.2) but on a significantly higher level. These data are outlined in Fig. 2.

A comparable observation was also made regarding the VAS leg. While there was no significant difference preoperatively between the PD and the RD group (62.9 and 65.5), this became highly significant in the 12 -month (17.0 vs 25.0$)$ and 24-month FU (15.6 vs 26.8), but still, also the RD group data demonstrated an obvious, sustainable and significant improvement. The graphical representation of these results is shown in Fig. 3.

Within the scope of this patient survey, the question of individual satisfaction was also asked. In the PD group, $71.7 \%$ stated that they were fully content 12 months after

VAS Back mean \pm SEM

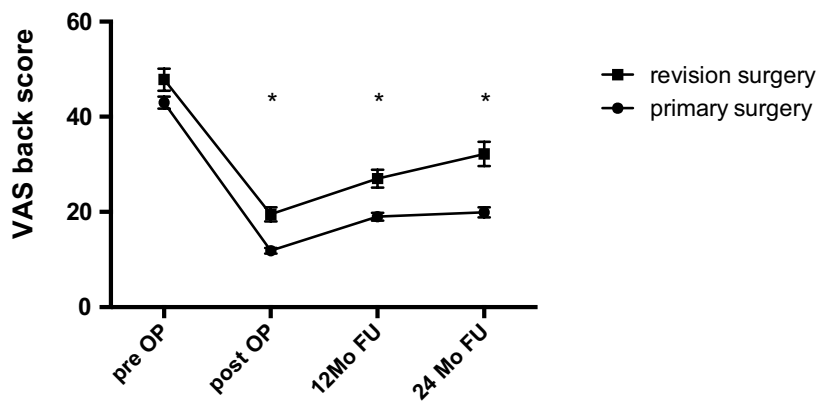

Fig. 2 Preoperative and postoperative course of the VAS back pain for primary versus revision surgery up to 24 month follow up

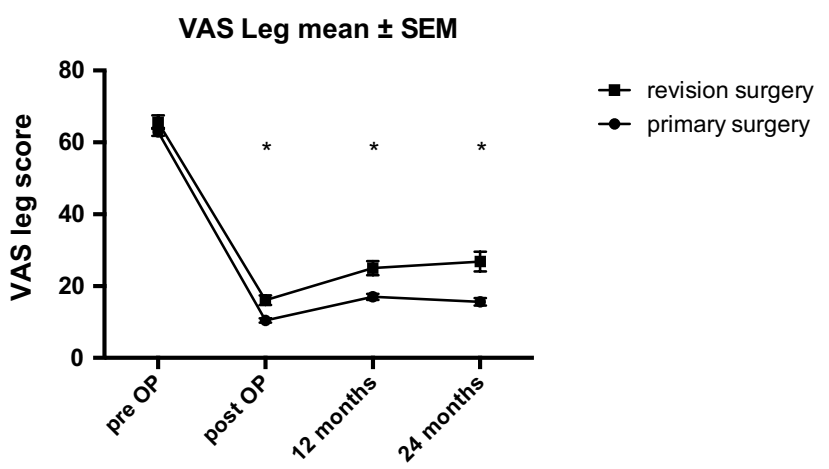

Fig. 3 Preoperative and postoperative course of the VAS leg pain for primary versus revision surgery up to 24 month follow up 
the operation and $22.6 \%$ were rather content with the result of the operation. In the RD group, a slightly worse, but still acceptable postoperative result was also achieved in this category ( $>80 \%$ contentment). In $55.8 \%$ of the cases, a full content was given and in $25.8 \%$, a rather content was given. This graphic representation is shown in Fig. 4.

During the primary interventions, a dura lesion occurred in $1.5 \%$ of the cases $(n=11 / 894)$, during revision interventions in $7.7 \%$ of the cases $(n=9 / 117)$.

\section{Discussion}

In principle, the clinical outcome data after an intervertebral disc operation can be described as good, regardless of the technique used [5-9]. The reasons for a recurrency of a lumbar disc herniation are multifactorial. In a number of studies, potential risk factors have been identified. Gender (male), smoking and heavy work seem to be independent risk factors [10-13]. However, the individual genetic predisposition with ongoing or accelerated degeneration of the disc seemed to be the decisive factor in the development of recurrences. It influences the competence of the disc annulus and also the

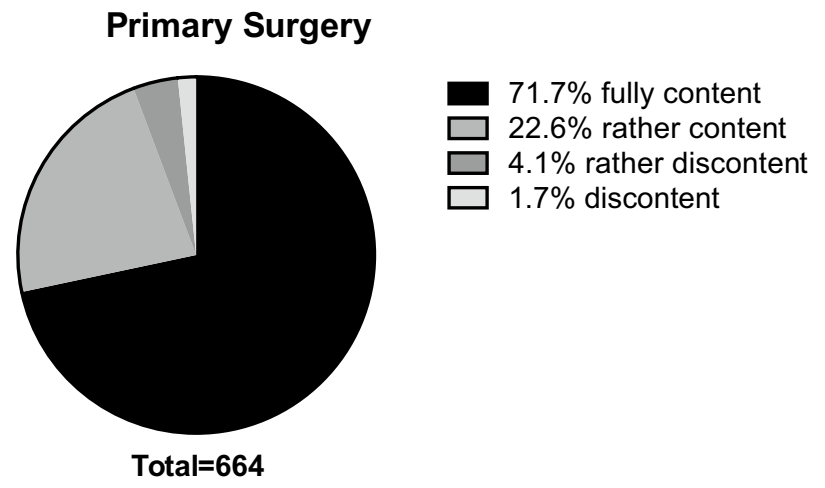

Revision Surgery

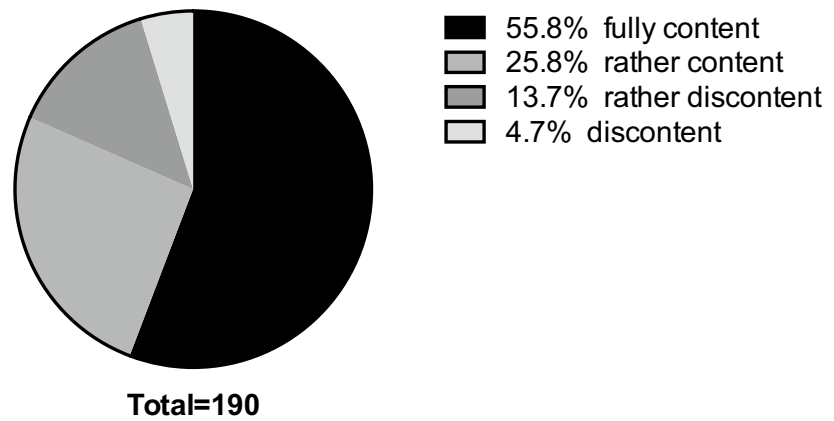

Fig. 4 Individual satisfaction 12 month after primary surgery and revision surgery morphology of the herniated disc. Kim et al. showed that the disc height and the sagittal range of motion of the index segment had a significant correlation with higher recurrence rates [14]. Carragee et al. reported that the lowest rate of reherniation and reoperation (1\%) with the best clinical results can be assumed for sequestered disc herniations with a small annular defect followed by contained, fragmented disc herniations with a small annular tear (10\%) [15]. Furthermore, patients with extruded fragments and massive posterior annulus defect have a significantly increased risk of recurrence $(27 \%)$ and reoperation $(21 \%)$. In case of contained non-fragmented herniations, $38 \%$ of the patients had recurrent or persistent sciatica.

Our clinical data prove that a pure microsurgical rediscectomy delivers satisfactory clinical results even in a high percentage of cases. Even if these results are slightly inferior compared to those of primary surgery, they can still be described as a successful target-oriented surgical technique. Of course, it is obvious in this cohort that the clinical symptom of back pain is rather subordinate preoperatively, but that there is nevertheless a significant improvement in this symptom postoperatively. This can be explained by the pressure relief of the posterior longitudinal ligament and possibly also of the dorsal annulus.

The role of limited (sequestrectomy) versus aggressive disc removal (discectomy) with regard to the postoperative results is still controversially discussed in the literature. McGrit et al. reported about a lower recurrence rate in aggressive discectomy in contrast to pure sequestrectomy in a large systematic review [16]. In contrast, the clinical results 2 years postoperative regarding back pain are significantly worse in the aggressive discectomy group than in the sequestrectomy group. Thome et al. came to a different result in a prospective, randomized study with the same question [7]. In this study, no difference in the recurrence rate was found between the two groups. However, the agreement in the observation of the development of back pain in the further FU in the aggressive discectomy group, which was published in a further investigation of the same cohorts by Barth et al., is striking [8].

Thus, in our opinion, it is advisable to adapt the surgical technique to the individual conditions of the respective findings. In case of a free sequester, a pure sequestrectomy is certainly the most minimally invasive option, with good clinical long-term results and low recurrence rates. Both in primary and recurrent surgery, a more aggressive exploration of the disc space with the removal of loose intradiscal disc fragments is indicated, if the disc space is high and the defect in the annulus large, which corresponds to a high risk of a recurrent disc herniation.

In Fig. 5a, typical case from everyday life is demonstrated which leaves, depending on the clinical symptoms and expectations of the patient, all possibilities for 
Fig. 5 Example of 33 year-old female patient with recurrent disc herniation L5/S1 with leg pain and severe low back pain treated with anterior rediscectomy and implantation of a total lumbar disc replacement. a Initial MRI with central disc herniation. b MRI of the recurrent disc herniation at the same location after microsurgical discectomy L5/S1. c Final result after anterior re-discectomy and implantation of a total lumbar disc replacement

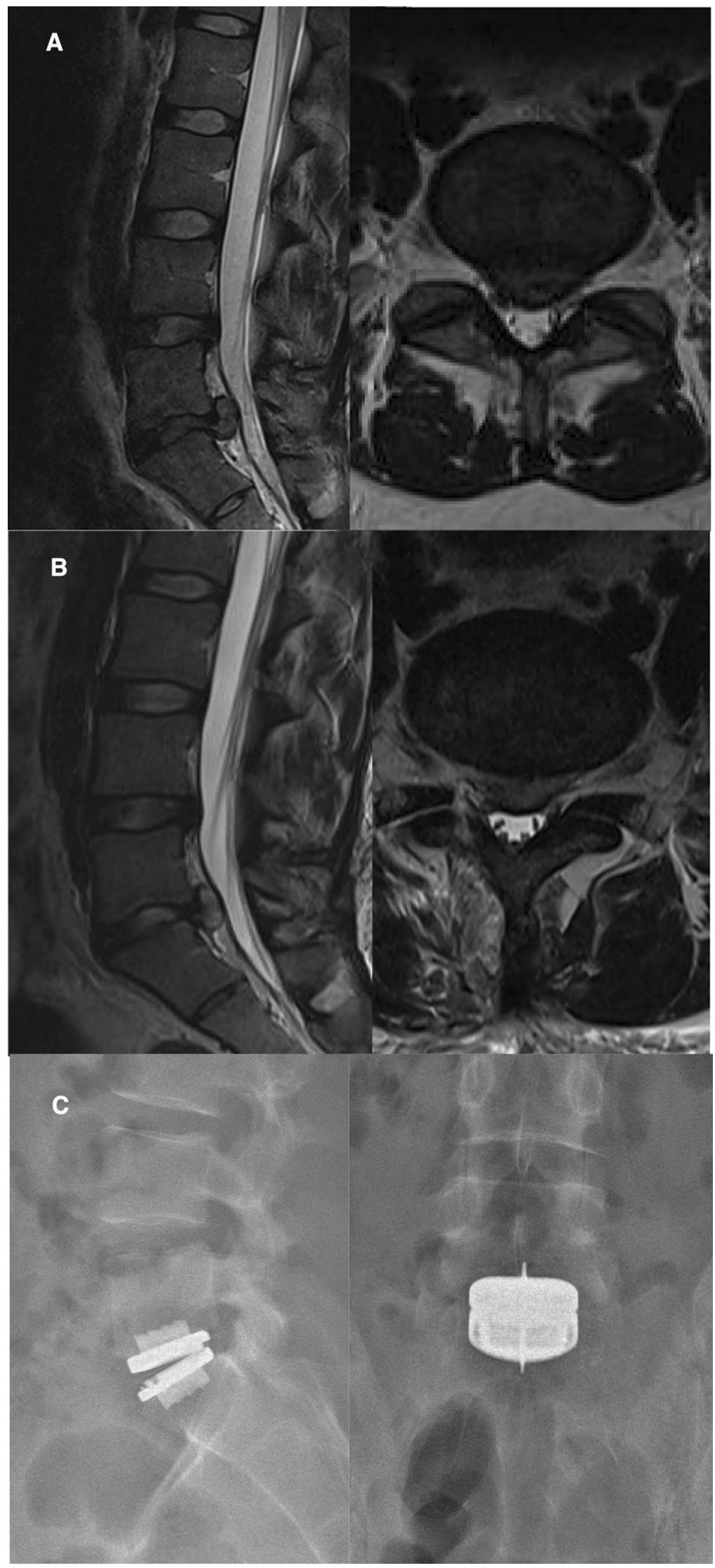


Fig. 6 Example of 69 year-old male patient with the third recurrent disc herniation L3/4 with leg pain and severe low back pain treated finally with posterior re-discectomy and fusion L3/4 including TLIF procedure with screw-rod-instrumentation. a Initial left-sided medio-lateral/intraforaminal disc herniation. b Right-sided mediolateral recurrency. c Second recurrent disc herniation. d Third central located recurrent disc herniation. e Final result after revision surgery

further surgical procedures open. This is a 33-year-old female who, as shown in picture A, initially received the indication for microsurgical discectomy from the right due to leading right-sided leg pain. After a symptom-free postoperative interval of 6 months, renewed complaints with now also clear back pain occurred. The MRT shows a fresh re-sequester (B) in a similar localization with a slight loss of the intervertebral disc height. Risk factors for further recurrence after another minimally invasive surgery are certainly the young age of the patient with corresponding activity and mobility of the disc, the size of the central annulus lesion and the non-fragmented contained sequester. After discussion of all chances and risks, the patients decided to have a total mobile disc replacement as shown in $\mathrm{C}$ postoperatively. Within this option, the integrity of the facet joints as well as the intraspinal scarring after the first intervention must be critically examined. Patients with far sequestrated fragments are also not suitable for this purely ventral motion-preserving procedure.

\section{When is additional stabilization necessary?}

Repetitively, the question arises when a fusion is necessary or justified in the case of a recurrent herniated disc. A mere number of previous operations cannot answer this question. Usually, the history and the complaint of the patient and especially the radiological diagnostics lead to the corresponding answer. This is also demanded several times as a conclusion in different review studies and supports the own experiences [13, 17-20].

If the patient is suffering from significant low back pain prior to the planned operation or if radiologically a severe instability of the functional spinal unit is proven, microsurgical decompression of the spinal canal should be combined with a stabilizing procedure. Also an extremely high probability of recurrence in combination with the patient's unwillingness to take this risk of a new herniated disc, justifies a "final" solution with fusion of the mobile segment. Via a pedicle screw-rod system, the physiologic alignment of the vertebral bodies can be restored. The decompression of the neurological structures takes place in an analogous microsurgical technique. Due to stability reasons, we favor a $360^{\circ}$ fusion,
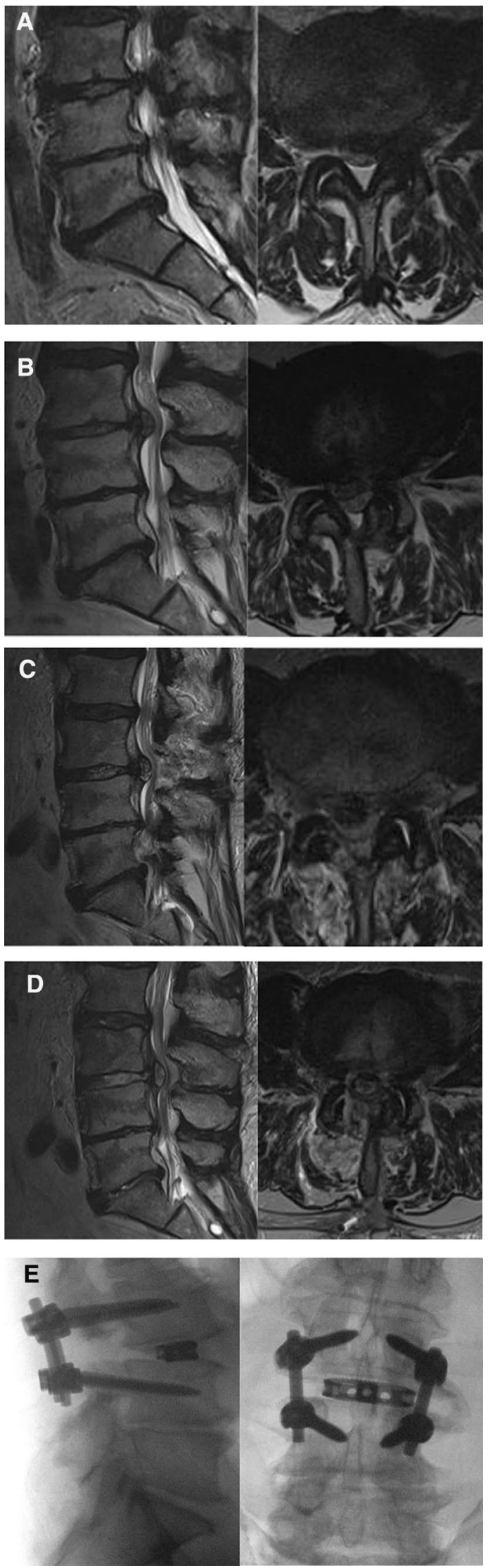
which can be achieved via a PLIF or TLIF cage, but also in case of a very high disc space or severe instabilities via an anterior retroperitoneal approach with an ALIF or OLIF procedure. Due to the circumstances in this indication spectrum that you have to decompress the spinal canal and explore the disc space from dorsal anyway, a posterior intervertebral fusion procedure volunteers. As an example, Fig. 6 shows a case with a final fusion after 3 microsurgical interventions within 4 years.

\section{Conclusion}

In summary, on the basis of our experience and the results described in the literature, it can be stated that there is no clear guideline for the surgical treatment of recurrent disc herniations. In most cases, a pure re-discectomy is sufficient and can be performed safely and effectively with the help of a microscope. Depending on the clinical and radiological findings, a simultaneous fusion with a re-discectomy in the index segment is indicated in the case of severe preoperative back pain with clear radiological signs of disc degeneration or proven instability. In principle, good clinical results can be achieved with revision indications, but with inferior results compared to primary interventions.

Acknowledgements Open access funding provided by Paracelsus Medical University.

Funding The authors did not receive any outside funding or grants in support of their research or preparation of this work.

\section{Compliance with ethical standards}

Conflict of interest The authors certifies that they have no commercial associations that might pose a conflict of interest in connection with this article.

Open Access This article is distributed under the terms of the Creative Commons Attribution 4.0 International License (http://creativeco mmons.org/licenses/by/4.0/), which permits unrestricted use, distribution, and reproduction in any medium, provided you give appropriate credit to the original author(s) and the source, provide a link to the Creative Commons license, and indicate if changes were made.

\section{References}

1. Weinstein JN, Lurie JD, Tosteson TD et al (2006) Surgical vs nonoperative treatment for lumbar disk herniation: the Spine Patient Outcomes Research Trial (SPORT) observational cohort. JAMA 296:2451-2459. https://doi.org/10.1001/jama.296.20.2451

2. Wang Y, Liang Z, Wu J et al (2019) Comparative clinical effectiveness of tubular microdiscectomy and conventional microdiscectomy for lumbar disc herniation: a systematic review and network meta-analysis. Spine (Phila Pa 1976). https://doi. org/10.1097/brs.0000000000003001
3. Overdevest GM, Peul WC, Brand R et al (2017) Tubular discectomy versus conventional microdiscectomy for the treatment of lumbar disc herniation: long-term results of a randomised controlled trial. J Neurol Neurosurg Psychiatry 88:1008-1016. https ://doi.org/10.1136/jnnp-2016-315306

4. Lebow RL, Adogwa O, Parker SL et al (2011) Asymptomatic same-site recurrent disc herniation after lumbar discectomy: results of a prospective longitudinal study with 2-year serial imaging. Spine (Phila Pa 1976) 36:2147-2151. https://doi.org/10.1097/ brs.0b013e3182054595

5. Ruetten S, Komp M, Merk H, Godolias G (2008) Full-endoscopic interlaminar and transforaminal lumbar discectomy versus conventional microsurgical technique: a prospective, randomized, controlled study. Spine (Phila Pa 1976) 33:931-939. https://doi. org/10.1097/brs.0b013e31816c8af7

6. Ruetten S, Komp M, Merk H, Godolias G (2009) Recurrent lumbar disc herniation after conventional discectomy: a prospective, randomized study comparing full-endoscopic interlaminar and transforaminal versus microsurgical revision. J Spinal Disord Tech 22:122-129. https://doi.org/10.1097/BSD.0b013e318175ddb4

7. Thome C, Barth M, Scharf J, Schmiedek P (2005) Outcome after lumbar sequestrectomy compared with microdiscectomy: a prospective randomized study. J Neurosurg Spine 2:271-278. https ://doi.org/10.3171/spi.2005.2.3.0271

8. Barth M, Diepers M, Weiss C, Thome C (2008) Two-year outcome after lumbar microdiscectomy versus microscopic sequestrectomy: part 2: radiographic evaluation and correlation with clinical outcome. Spine (Phila Pa 1976) 33:273-279. https://doi. org/10.1097/brs.0b013e31816201a6

9. Arts M, Brand R, van der Kallen B et al (2011) Does minimally invasive lumbar disc surgery result in less muscle injury than conventional surgery? A randomized controlled trial. Eur spine J Off Publ Eur Spine Soc Eur Spinal Deform Soc Eur Sect Cerv Spine Res Soc 20:51-57. https://doi.org/10.1007/s00586-010-1482-y

10. Shimia M, Babaei-Ghazani A, Sadat BE et al (2013) Risk factors of recurrent lumbar disk herniation. Asian J Neurosurg 8:93-96. https://doi.org/10.4103/1793-5482.116384

11. Ambrossi GL, McGirt MJ, Sciubba DM et al (2009) Recurrent lumbar disc herniation after single-level lumbar discectomy: incidence and health care cost analysis. Neurosurgery 65:574-578. https://doi.org/10.1227/01.neu.0000350224.36213.f9 (discussion 578)

12. Miwa S, Yokogawa A, Kobayashi T et al (2013) Risk factors of recurrent lumbar disc herniation: a single center study and review of the literature. J Spinal Disord Tech. https://doi.org/10.1097/ BSD.0b013e31828215b3

13. Shepard N, Cho W (2019) Recurrent lumbar disc herniation: a review. Glob spine J 9:202-209. https://doi.org/10.1177/21925 68217745063

14. Kim KT, Park SW, Kim YB (2009) Disc height and segmental motion as risk factors for recurrent lumbar disc herniation. Spine (Phila Pa 1976) 34:2674-2678. https://doi.org/10.1097/brs.0b013 e3181b4aaac

15. Carragee EJ, Han MY, Suen PW, Kim D (2003) Clinical outcomes after lumbar discectomy for sciatica: the effects of fragment type and anular competence. J Bone Jt Surg Am 85-A:102-108

16. McGirt MJ, Ambrossi GL, Datoo G et al (2009) Recurrent disc herniation and long-term back pain after primary lumbar discectomy: review of outcomes reported for limited versus aggressive disc removal. Neurosurgery 64:335-338. https://doi. org/10.1227/01.neu.0000337574.58662.e2

17. Hlubek RJ, Mundis GMJ (2017) Treatment for recurrent lumbar disc herniation. Curr Rev Musculoskelet Med 10:517-520. https ://doi.org/10.1007/s12178-017-9450-3 
18. Onyia CU, Menon SK (2017) The debate on most ideal technique for managing recurrent lumbar disc herniation: a short review. Br J Neurosurg 31:701-708. https://doi.org/10.1080/02688 697.2017.1368451

19. Ajiboye RM, Drysch A, Mosich GM et al (2018) Surgical treatment of recurrent lumbar disk herniation: a systematic review and meta-analysis. Orthopedics 41:e457-e469. https://doi. org/10.3928/01477447-20180621-01
20. Dower A, Chatterji R, Swart A, Winder MJ (2016) Surgical management of recurrent lumbar disc herniation and the role of fusion. J Clin Neurosci Off J Neurosurg Soc Australas 23:44-50. https:// doi.org/10.1016/j.jocn.2015.04.024

Publisher's Note Springer Nature remains neutral with regard to jurisdictional claims in published maps and institutional affiliations. 